\title{
Variational Ritz Method for the Elastic Stress Analysis of Plates under Uniaxial Parabolic Distributed Edge Loads
}

\author{
Nwoji, C.U1 ., Ike, C.C. ${ }^{* 2}$, Onah, H.N ${ }^{3}$, Mama, B.O. ${ }^{4}$ \\ ${ }^{1}$ Dept of Civil Engineering University of Nigeria, Nsukka, Enugu State, Nigeria. \\ ${ }^{2}$ Dept of Civil Engineering Enugu State University of Science \& Technology, Enugu State, Nigeria. \\ ${ }^{3}$ Dept of Civil Engineering University of Nigeria, Nsukka, Enugu State, Nigeria. \\ ${ }^{4}$ Dept of Civil EngineeringUniversity of Nigeria, Nsukka, Enugu State, Nigeria.
}

\begin{abstract}
In this work, the variational Ritz method was formulated and applied to elastic stress analysis of rectangular plates $(2 a \times 2 b)$ under parabolically distributed edge loads applied at the two faces $x= \pm a$.

The problem solved is a classical two dimensional problem of elasticity that can be solved by finding solutions of the biharmonic problem of Airy's stress function which satisfy the biharmonic problem in closed form on the plate domain and simultaneously satisfy the stress boundary conditions. Analytical solutions of this problem are difficult to obtain, hence the numerical solution presented in this study. The variational formulation used energy principles and assumed the plate is in plane stress state. The Ritz method was then used to obtain the first variation of the total energy function which represents the equilibrium state of the plate under the applied load. One term Airy's stress function and three term Airy's stress functions were used to solve the Ritz variational equation, and thus obtain solutions for the unknown parameters of the Airy's stress potential functions. The normal and shear stress fields were then determined. The solutions obtained for the normal and shear stress fields were found to satisfy all the stress boundary conditions along all the edges $x= \pm a, y= \pm b$ of the plate as well as the governing equations of the problem. The Ritz variational solutions of the normal and shear stress fields were in agreement with solutions obtained in literature.
\end{abstract}

Keywords: Variational Ritz method, stress fields, biharmonic problem, Ritz variational equation, Airy's stress potential function, stress boundary conditions, plane stress elasticity problem, total energy functional.

\section{Introduction}

Thin rectangular plates subjected to non-uniformly distributed edge loads are very common in engineering applications as components of aircraft panels, spacecraft panels and machine panels. Accurate determination of the stress distribution in such plates is very vital for the elastic design of such structures. Due to the complex nature of such problems, no mathematically exact or analytical closed form solution has been given so far for thin rectangular plates under non-uniform in plane distributed edge loads (Tang and Wang, 2011). Tang and Wang adopted Chebyshev polynomials as the stress function which satisfy the stress boundary conditions, and then used Ritz method to find the distribution of inplane stresses of thin rectangular plates under non linearly distributed edge loads based on the theory of elasticity. They studied plates with different aspect ratios under uniaxial and biaxial parabolic edge compressions with the help of the mathematical computational software Mathematica (Tang and Wang, 2011). Their solutions satisfy exactly the stress boundary conditions, and agree excellently with numerical results obtained by the finite element method and differential quadrature method (Tang and Wang, 2011). The plane elasticity problem for thin isotropic plates consists of obtaining solutions to the biharmonic equation in terms of Airy's stress function (Devarakonda, 2004) as given by:

$$
\left(\frac{\partial^{4}}{\partial x^{4}}+\frac{2 \partial^{4}}{\partial x^{2} \partial y^{2}}+\frac{\partial^{4}}{\partial y^{4}}\right) \phi(x, y)=0
$$

where $\phi(x, y)$ is the Airy's stress function

The stresses are determined from $\phi(x, y)$ by

$$
\sigma_{x x}=\frac{\partial^{2} \phi}{\partial y^{2}} \quad \sigma_{y y}=\frac{\partial^{2} \phi}{\partial x^{2}} \quad \tau_{x y}=-\frac{\partial^{2} \phi}{\partial x \partial y}
$$

where $\sigma_{x x}, \sigma_{y y}$ are normal stresses, and $\tau_{x y}$ is the shear stress.

\footnotetext{
${ }^{*}$ Corresponding author
} 


\section{Research aim and objectives}

The general aim and objective of this study is to apply the variational Ritz method to solve the elasticity problem of finding stresses in rectangular plates under inplane loads distributed parabolically on the two faces $(x= \pm a)$ of the plate. The specific objectives are:

(i) to formulate the problem as a variational problem by formulating the variational functional for the problem.

(ii) to simplify the variational functional formulated using Airy's stress function, $\phi(x, y)$.

(iii) to solve the variational problem formulated in Airy's stress function to obtain the Airy stress function that ensures equilibrium of the problem.

(iv) to find the stress fields $\sigma_{x x}, \sigma_{y y}$, and $\tau_{x y}$ from the Airy's stress function.

\section{Research methodology / theoretical framework}

For two dimensional linear elasticity problems on the $x y$ coordinate plane, the total potential energy functional, $\Pi$ is given by Equation (2): (Dixit, 2007):

$$
\Pi=U=\frac{1}{2} \iint_{R}\left(\sigma_{x x} \varepsilon_{x x}+\sigma_{y y} \varepsilon_{y y}+\tau_{x y} \gamma_{x y}\right) d x d y
$$

where $U=$ strain energy; $\sigma_{x x}, \sigma_{y y}$ are normal stresses; $\tau_{x y}$ is the shear stress, $\varepsilon_{x x}, \varepsilon_{y y}$ are normal strains, $\gamma_{x y}$ is the shear strain and $R$ is the domain of definition of the two dimensional elastic body.

For plane stress problems, the stress-strain law can be expressed as Equations (3-8)

$$
\begin{aligned}
\varepsilon_{x x} & =\frac{1}{E}\left(\sigma_{x x}-\mu \sigma_{y y}\right) \\
\varepsilon_{y y} & =\frac{1}{E}\left(\sigma_{y y}-\mu \sigma_{x x}\right) \\
\gamma_{x y} & =\frac{\tau_{x y}}{G}=\frac{2(1+\mu) \tau_{x y}}{E} \\
\sigma_{z z} & =0 \\
\tau_{x z} & =0 \\
\tau_{y z} & =0
\end{aligned}
$$

where $G=\frac{E}{2(1+\mu)}$

$G$ is the shear modulus, $E$ is the Young's modulus, $\mu$ is the Poisson's ratio

For plane strain conditions, the stress-strain law can be expressed as

$$
\begin{aligned}
& \varepsilon_{x x}=\frac{1-\mu^{2}}{E}\left(\sigma_{x x}-\frac{\mu}{1-\mu} \sigma_{y y}\right) \\
& \varepsilon_{y y}=\frac{1-\mu^{2}}{E}\left(\sigma_{y y}-\frac{\mu}{1-\mu} \sigma_{x x}\right) \\
& \gamma_{x y}=\frac{2(1+\mu)}{E} \tau_{x y}=\frac{\tau_{x y}}{G}
\end{aligned}
$$

Using the stress-strain law in the total potential energy functional, we express the total potential energy functional in terms of stresses as

$$
\begin{gathered}
\Pi=U=\frac{1}{2} \iint_{R}\left[\sigma_{x x} \frac{1}{E}\left(\sigma_{x x}-\mu \sigma_{y y}\right)+\sigma_{y y} \frac{1}{E}\left(\sigma_{y y}-\mu \sigma_{x x}\right)+\tau_{x y}\left(\frac{2(1+\mu)}{E}\right) \tau_{x y}\right] d x d y \\
\Pi=\frac{1}{2 E} \iint_{R}\left[\sigma_{x x}^{2}+\sigma_{y y}^{2}-2 \mu \sigma_{x x} \sigma_{y y}+2(1+\mu) \tau_{x y}^{2}\right] d x d y \\
\delta \Pi=0
\end{gathered}
$$




$$
\iint_{R}\left(\sigma_{x x} \delta \sigma_{x x}+\sigma_{y y} \delta \sigma_{y y}+2 \tau_{x y} \delta \tau_{x y}\right) d x d y=0
$$

We recall that for conservative body force fields, the inplane stress fields of two dimensional elasticity problems are derivable from Airy's stress functions $\phi(x, y)$ as follows

$$
\begin{aligned}
& \sigma_{x x}=\frac{\partial^{2} \phi}{\partial y^{2}}+V \\
& \sigma_{y y}=\frac{\partial^{2} \phi}{\partial x^{2}}+V \\
& \tau_{x y}=-\frac{\partial^{2} \phi}{\partial x \partial y}
\end{aligned}
$$

where $F_{x}=-\frac{\partial V}{\partial x}$

$$
\begin{aligned}
& F_{y}=-\frac{\partial V}{\partial y} \\
& \vec{F}=-\frac{\partial V}{\partial x} i-\frac{\partial V}{\partial y} j \\
& \vec{F}=-\nabla \cdot V=-\operatorname{grad} V
\end{aligned}
$$

In the absence of body forces,

$$
\begin{gathered}
\sigma_{x x}=\frac{\partial^{2} \phi}{\partial y^{2}} \\
\sigma_{y y}=\frac{\partial^{2} \phi}{\partial x^{2}} \\
\tau_{x y}=-\frac{\partial^{2} \phi}{\partial x \partial y}
\end{gathered}
$$

The total potential energy functional becomes expressed in terms of the Airy's stress function $\phi(x, y)$ as

$$
\Pi=\frac{1}{2 E} \iint\left[\left(\frac{\partial^{2} \phi}{\partial x^{2}}\right)^{2}+\left(\frac{\partial^{2} \phi}{\partial y^{2}}\right)^{2}-2 \mu \frac{\partial^{2} \phi}{\partial x^{2}} \frac{\partial^{2} \phi}{\partial y^{2}}+2(1+\mu)\left(-\frac{\partial^{2} \phi}{\partial x \partial y}\right)^{2}\right] d x d y
$$

Let $\phi(x, y)=\phi_{0}+\sum_{i=1}^{\infty} c_{i} \phi_{i}$

Then for arbitrary variation of the unknown parameters $c_{i}$ of the Airy's stress function, the variation in $\Pi$ is

$$
\begin{gathered}
\delta \Pi=\sum \delta c_{i} \iint\left[\frac{\partial^{2} \phi}{\partial y^{2}} \frac{\partial^{2} \phi_{i}}{\partial y^{2}}+\frac{\partial^{2} \phi}{\partial x^{2}} \frac{\partial^{2} \phi_{i}}{\partial x^{2}}-2 \mu \frac{\partial \phi}{\partial x} \frac{\partial \phi_{i}}{\partial x} \frac{\partial \phi}{\partial y} \frac{\partial \phi_{i}}{\partial y}\right. \\
\left.+(2+2 \mu) \frac{\partial^{2} \phi}{\partial x \partial y} \frac{\partial^{2} \phi_{i}}{\partial x \partial y}\right] d x d y=0 \\
\sum \delta c_{i} \iint\left[\frac{\partial^{2} \phi}{\partial y^{2}} \frac{\partial^{2} \phi_{i}}{\partial y^{2}}+\frac{\partial^{2} \phi}{\partial x^{2}} \frac{\partial^{2} \phi_{i}}{\partial x^{2}}-2 \mu \frac{\partial^{2} \phi}{\partial x \partial y} \frac{\partial^{2} \phi_{i}}{\partial x \partial y}+2 \frac{\partial^{2} \phi}{\partial x \partial y} \frac{\partial^{2} \phi_{i}}{\partial x \partial y}\right. \\
\left.+2 \mu \frac{\partial^{2} \phi}{\partial x \partial y} \frac{\partial^{2} \phi_{i}}{\partial x \partial y}\right] d x d y=0
\end{gathered}
$$




$$
\sum \delta c_{i} \iint_{R}\left[\frac{\partial^{2} \phi}{\partial y^{2}} \frac{\partial^{2} \phi_{i}}{\partial y^{2}}+\frac{\partial^{2} \phi}{\partial x^{2}} \frac{\partial^{2} \phi_{i}}{\partial x^{2}}+2 \frac{\partial^{2} \phi}{\partial x \partial y} \frac{\partial^{2} \phi_{i}}{\partial x \partial y}\right] d x d y=0
$$

For $N$ arbitrary variations of the unknown parameters of the Airy's stress function, the first variation of the total energy functional yields a system of $N$ equations in terms of $c_{i}, i=1,2, \ldots, N$ given by Equation (30).

\section{Application of Ritz method to a plate under uniaxial parabolic inplane load}

As a specific illustration of the application of the variational Ritz method, consider the rectangular thin plate $2 a \times 2 b$ loaded in plane as shown in Figure 1. The origin of the Cartesian coordinates system is chosen to be the center of the plate in order to take advantage of the symmetry of the plate and the symmetrical nature of the load distribution. The $x$ and $y$ coordinate axes are chosen to be parallel to the edges as shown in Figure 1 .

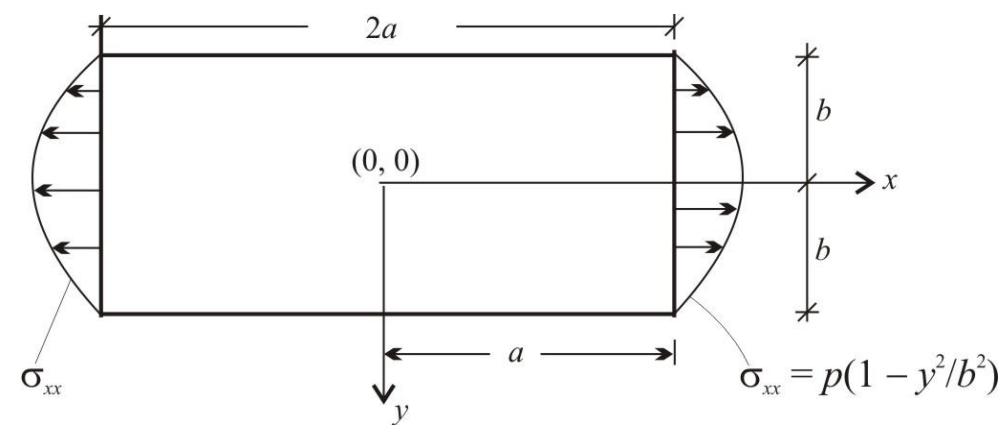

Figure 1: Rectangular plate under distributed parabolic edge load in the $x$-direction

It is assumed that there are no body forces. The Ritz method is applied to determine the stress fields $\sigma_{x x}, \sigma_{y y}$ and $\tau_{x y}$ in the rectangular thin plate due to the distributed parabolic edge loads $\sigma_{x x}=p\left(1-\frac{y^{2}}{b^{2}}\right)$ acting on the plate faces $x=a, x=-a$. Since there are no body forces, $\vec{F}=0, F_{x}=0, F_{y}=0, F_{z}=0$. where $\vec{F}$ is the body force vector, and $F_{x}, F_{y}, F_{z}$ are the $x, y$, and $z$ components of the body force vector. The stress boundary conditions are given by:

$$
\sigma_{x x}(x= \pm a)=p\left(1-\frac{y^{2}}{b^{2}}\right)
$$

where $p$ is the value of $\sigma_{x x}(x= \pm a)$ when $y=0$.

$$
\begin{aligned}
& \tau_{x y}(x= \pm a)=0 \\
& \tau_{x y}(y= \pm b)=0 \\
& \sigma_{y y}(y= \pm b)=0
\end{aligned}
$$

The Airy's stress potential function $\phi(x, y)$ is approximated as a linear combination of coordinate shape (basis) functions such that

$$
\phi(x, y)=\phi_{0}(x, y)+\sum_{m=1}^{N} c_{m} \phi_{m}(x, y)
$$

where $\phi_{m}(x, y)$ are the coordinate shape(basis) functions, and $c_{m}$ are the $N$ undetermined parameters of the Airy's stress potential functions, and $\phi_{0}(x, y)$ is the Airy stress potential function that is chosen such that it satisfies the stress boundary conditions on the plate edges. $\phi_{m}(x, y)$ are coordinate functions chosen as to satisfy the stress conditions within the plate domain. $\phi_{0}(x, y)$ is chosen to satisfy the stress boundary conditions on $x= \pm a$, and $y= \pm b$. Using the definition of Airy's stress potential function, 


$$
\sigma_{x x}(x= \pm a)=p\left(1-\frac{y^{2}}{b^{2}}\right)=\frac{\partial^{2} \phi}{\partial y^{2}}(x, y)
$$

Integrating,

$$
\frac{\partial \phi_{0}}{\partial y}=p y-\frac{p y^{3}}{3 b^{2}}+c_{1}
$$

where $c_{1}$ is a constant of integration.

Integrating again,

$$
\phi_{0}(x, y)=\frac{p y^{2}}{2}-\frac{p y^{4}}{12 b^{2}}+c_{1} y+c_{2}
$$

where $c_{2}$ is a constant of integration

$$
\sigma_{y y}^{0}=\frac{\partial^{2} \phi_{0}}{\partial x^{2}}=0
$$

where $\sigma_{y y}^{0}$ is the normal stress on the edge $y= \pm b$

$$
\tau_{x y}^{0}=-\frac{\partial^{2} \phi}{\partial x \partial y}=0
$$

$\tau_{x y}^{0}$ is the shear stress on the edges $x= \pm a, y= \pm b$.

Hence, $c_{1}=c_{2}=0$

Thus $\phi_{0}(x, y)$ becomes:

$$
\phi_{0}(x, y)=\frac{p y^{2}}{2}-\frac{p y^{4}}{12 b^{2}}=\frac{p y^{2}}{2}\left(1-\frac{y^{2}}{6 b^{2}}\right)
$$

$\phi_{m}(x, y)$ is chosen to satisfy the zero traction boundary conditions on the edges $x= \pm a, y= \pm b$ and such that it qualifies as a suitable Airy's stress potential function. Hence we choose

$$
\begin{aligned}
& \phi_{1}(x, y)=\left(x^{2}-a^{2}\right)^{2}\left(y^{2}-b^{2}\right)^{2} \\
& \phi_{2}(x, y)=x^{2}\left(x^{2}-a^{2}\right)^{2}\left(y^{2}-b^{2}\right)^{2} \\
& \phi_{3}(x, y)=y^{2}\left(y^{2}-b^{2}\right)^{2}\left(x^{2}-a^{2}\right)^{2}
\end{aligned}
$$

Then

$$
\phi(x, y)=\frac{p y^{2}}{2}\left(1-\frac{y^{2}}{6 b^{2}}\right)+\left(x^{2}-a^{2}\right)^{2}\left(y^{2}-b^{2}\right)^{2}\left(c_{1}+c_{2} x^{2}+c_{3} y^{2}+\ldots\right)
$$

A one parameter choice of the stress potential function is thus

$$
\phi(x, y)=\frac{p y^{2}}{2}\left(1-\frac{y^{2}}{6 b^{2}}\right)+c_{1}\left(x^{2}-a^{2}\right)^{2}\left(y^{2}-b^{2}\right)^{2}
$$

The Ritz variation equation is thus

$$
\iint\left[\frac{\partial^{2} \phi}{\partial y^{2}} \frac{\partial^{2} \phi_{1}}{\partial y^{2}}+\frac{\partial^{2} \phi}{\partial x^{2}} \frac{\partial^{2} \phi_{1}}{\partial x^{2}}+2 \frac{\partial^{2} \phi}{\partial x \partial y} \frac{\partial^{2} \phi_{1}}{\partial x \partial y}\right] d x d y=0
$$

where $\phi_{1}=\left(x^{2}-a^{2}\right)^{2}\left(y^{2}-b^{2}\right)^{2}$

$$
\begin{aligned}
& \frac{\partial \phi_{1}}{\partial x}=4\left(x^{3}-x a^{2}\right)\left(y^{2}-b^{2}\right)^{2} \\
& \frac{\partial^{2} \phi_{1}}{\partial x^{2}}=4\left(3 x^{2}-a^{2}\right)\left(y^{2}-b^{2}\right)^{2}
\end{aligned}
$$




$$
\begin{aligned}
& \frac{\partial^{2} \phi_{1}}{\partial y^{2}}=4\left(3 y^{2}-b^{2}\right)\left(x^{2}-a^{2}\right)^{2} \\
& \frac{\partial^{2} \phi_{1}}{\partial x \partial y}=\frac{\partial^{2} \phi_{1}}{\partial y \partial x}=16\left(x^{3}-x a^{2}\right)\left(y^{3}-y b^{2}\right) \\
& \phi=\frac{p}{2}\left(y^{2}-\frac{y^{4}}{6 b^{2}}\right)+c_{1}\left(x^{2}-a^{2}\right)^{2}\left(y^{2}-b^{2}\right)^{2} \\
& \frac{\partial \phi}{\partial y}=\frac{p}{2}\left(2 y-\frac{4 y^{3}}{6 b^{2}}\right)+c_{1}\left(4\left(y^{3}-y b^{2}\right)\left(x^{2}-a^{2}\right)^{2}\right) \\
& \frac{\partial^{2} \phi}{\partial y^{2}}=p\left(1-\frac{y^{2}}{b^{2}}\right)+4 c_{1}\left(3 y^{2}-b^{2}\right)\left(x^{2}-a^{2}\right)^{2} \\
& \frac{\partial^{2} \phi}{\partial x^{2}}=4 c_{1}\left(3 x^{2}-a^{2}\right)\left(y^{2}-b^{2}\right)^{2} \\
& \frac{\partial^{2} \phi}{\partial x \partial y}=16 c_{1}\left(y^{3}-y b^{2}\right)\left(x^{3}-x a^{2}\right)
\end{aligned}
$$

The region $R^{2}$ of integration is the two dimensional domain of the plate, given by $-a \leq x \leq a ;-b \leq y \leq b$ Thus, the Ritz variational integral is given by

$$
\begin{aligned}
& \int_{-a}^{a} \int_{-b}^{b}\left\{\left[p\left(1-\frac{y^{2}}{b^{2}}\right)+c_{1} 4\left(3 y^{2}-b^{2}\right)\left(x^{2}-a^{2}\right)^{2}\right] \cdot 4\left(3 y^{2}-b^{2}\right)\left(x^{2}-a^{2}\right)^{2}\right. \\
& +4 c_{1}\left(3 x^{2}-a^{2}\right)\left(y^{2}-b^{2}\right)^{2} \cdot 4\left(3 x^{2}-a^{2}\right)\left(y^{2}-b^{2}\right)^{2} \\
& \left.+2 \times 16 c_{1}\left(y^{3}-y b^{2}\right)\left(x^{3}-x a^{2}\right) 16\left(y^{3}-y b^{2}\right)\left(x^{3}-x a^{2}\right)\right\} d x d y=0 \\
& c_{1} \int_{-a}^{a} \int_{-b}^{b} 16\left(3 y^{2}-b^{2}\right)^{2}\left(x^{2}-a^{2}\right)^{4} d x d y+c_{1} \int_{-a}^{a} \int_{-b}^{b} 16\left(3 x^{2}-a^{2}\right)^{2}\left(y^{2}-b^{2}\right)^{4} d x d y \\
& +c_{1} \int_{-a}^{a} \int_{-b}^{b} 2 \times 256\left(y^{3}-y b^{2}\right)^{2}\left(x^{3}-x a^{2}\right)^{2} d x d y \\
& =-\int_{-a-b}^{a} \int_{-a}^{b} p\left(1-\frac{y^{2}}{b^{2}}\right) 4\left(3 y^{2}-b^{2}\right)\left(x^{2}-a^{2}\right)^{4} d x d y \\
& c_{1}\left\{16 \int_{-b}^{b}\left(3 y^{2}-b^{2}\right) d y \int_{-a}^{a}\left(x^{2}-a^{2}\right)^{4} d x+16 \int_{-a}^{a}\left(3 x^{2}-a^{2}\right) d x \int_{-b}^{b}\left(y^{2}-b^{2}\right)^{4} d y\right. \\
& \left.+512 \int_{-a}^{a}\left(x^{3}-x a^{2}\right)^{2} d x \int_{-b}^{b}\left(y^{3}-y b^{2}\right)^{2} d y\right\} \\
& =-4 p \int_{-b}^{b}\left(1-\frac{y^{2}}{b^{2}}\right)\left(3 y^{2}-b^{2}\right) d y \int_{-a}^{a}\left(x^{2}-a^{2}\right)^{2} d x
\end{aligned}
$$

Using online Wolfram integration software, we obtain

$$
\int_{-a}^{a}\left(x^{2}-a^{2}\right)^{4} d x=\frac{256}{315} a^{9}
$$




$$
\begin{aligned}
& \int_{-a}^{a}\left(3 x^{2}-a^{2}\right)^{2} d x=\frac{8}{5} a^{5} \\
& \int_{-a}^{a}\left(x^{2}-a^{2}\right)^{2} d x=\frac{16}{15} a^{5} \\
& \int_{-a}^{a}\left(x^{3}-x a^{2}\right)^{2} d x=\frac{16}{105} a^{7} \\
& \int_{-b}^{b}\left(1-\frac{y^{2}}{b^{2}}\right)\left(3 y^{2}-b^{2}\right) d y=-\frac{8}{15} b^{3} \\
& \int_{-b}^{b}\left(y^{2}-b^{2}\right)^{4} d y=\frac{256}{315} b^{9} \\
& \int_{-b}^{b}\left(3 y^{2}-b^{2}\right)^{2} d y=\frac{8}{5} b^{5} \\
& \int_{-b}^{b}\left(y^{2}-b^{2}\right)^{2} d y=\frac{16}{15} b^{5} \\
& \int_{-b}^{b}\left(y^{3}-y b^{2}\right)^{2} d y=\frac{16}{105} b^{7}
\end{aligned}
$$

Hence,

$$
\begin{gathered}
c_{1}\left(16 \times \frac{8 b^{5}}{5} \times \frac{256 a^{9}}{315}+16 \times \frac{8 a^{5}}{5} \times \frac{256 b^{9}}{315}+2 \times 256 \times \frac{16 a^{7}}{105} \times \frac{16 b^{7}}{105}\right) \\
=-4 p\left(-\frac{8 b^{3}}{15}\right)\left(\frac{16 a^{5}}{15}\right) \\
c_{1}=\frac{p b^{3} a^{5}}{\frac{64}{7}\left(b^{5} a^{9}+a^{5} b^{9}\right)+\frac{256}{49} a^{7} b^{7}} \\
c_{1}=\frac{p /\left(b^{2} a^{4}\right)}{\frac{64}{7}\left(1+\left(\frac{b}{a}\right)^{4}\right)+\frac{256}{49}\left(\frac{b}{a}\right)^{2}}
\end{gathered}
$$

Let $b / a=\alpha$

$$
\begin{aligned}
& c_{1}=\frac{p \alpha^{-2} a^{-6}}{\frac{64}{7}\left(1+\alpha^{4}\right)+\frac{256}{49} \alpha^{2}} \\
& c_{1}=F_{1}(\alpha) p a^{-6} \\
& F_{1}(\alpha)=\frac{p \alpha^{-2}}{\frac{64}{7}\left(1+\alpha^{4}\right)+\frac{256}{49} \alpha^{2}}
\end{aligned}
$$


Thus,

$$
\phi(x, y)=\frac{p y^{2}}{2}\left(1-\frac{y^{2}}{6 b^{2}}\right)+F_{1}(\alpha) p a^{-6}\left(x^{2}-a^{2}\right)^{2}\left(y^{2}-b^{2}\right)^{2}
$$

The stresses are then obtained from the definition of Airy's stress potential function as:

$$
\begin{aligned}
& \sigma_{y y}=\frac{\partial^{2} \phi}{\partial x^{2}}=4 c_{1}\left(3 x^{2}-a^{2}\right)\left(y^{2}-b^{2}\right)^{2} \\
& \sigma_{y y}=-4 c_{1}\left(1-\frac{3 x^{2}}{a^{2}}\right)\left(1-\frac{y^{2}}{b^{2}}\right)^{2} a^{2} b^{4} \\
& \sigma_{y y}=-4 F_{1}(\alpha) p a^{-6}\left(1-\frac{3 x^{2}}{a^{2}}\right)\left(1-\frac{y^{2}}{b^{2}}\right)^{2} a^{2} b^{4} \\
& \sigma_{y y}=-4 F_{1}(\alpha)\left(1-\frac{3 x^{2}}{a^{2}}\right)\left(1-\frac{y^{2}}{b^{2}}\right)^{2} p a^{-4} b^{4} \\
& \sigma_{y y}=-4 F_{1}(\alpha)\left(1-\frac{3 x^{2}}{a^{2}}\right)\left(1-\frac{y^{2}}{b^{2}}\right)^{2} p\left(\frac{b^{4}}{a^{4}}\right) \\
& \sigma_{y y}(x=0, y)=-4 F_{1}(\alpha)\left(1-\frac{y^{2}}{b^{2}}\right)^{2} p \alpha^{4} \\
& \tau_{x y}(0,0)=0 \\
& \left.\sigma_{x x}=\frac{\partial^{2} \phi}{\partial y^{2}}=p\left(1-\frac{y^{2}}{b^{2}}\right)+4 F_{1}(\alpha)\left(\frac{y^{2}}{b^{2}}-1\right)\left(\frac{x^{2}}{a^{2}}-1\right) x y p \alpha^{2} a^{-2}-b^{2}\right)\left(x^{2}-a^{2}\right)^{2} \\
& \tau_{x y}=-16 F_{1}(\alpha)\left(\frac{y^{2}}{b^{2}}-1\right)\left(\frac{x^{2}}{a^{2}}-1\right) x y p b^{2} a^{-4} \\
& \sigma_{x x}(x=0, y=0)=-4 F_{1}(\alpha) p a^{4} \\
& \tau_{x y}=-\frac{\partial^{2} \phi}{\partial x \partial y}=-16 c_{1}\left(y^{3}-y b^{2}\right)\left(x^{3}-x a^{2}\right) \\
& \tau_{x y}=-16 c_{1}\left(\frac{y^{2}}{b^{2}}-1\right)\left(\frac{y^{2}}{a^{2}}-1\right)\left(\frac{y^{2}}{a^{2}}\right)\left(x y b^{2} a^{2}\right. \\
& \left.\tau^{2}-1\right) x y b^{2} a^{2} \\
& \tau^{2}
\end{aligned}
$$




$$
\sigma_{x x}=p\left(1-\frac{y^{2}}{b^{2}}\right)+4 F_{1}(\alpha) p a^{-6}\left(3 y^{2}-b^{2}\right)\left(x^{2}-a^{2}\right)^{2}
$$

For square plates, $\alpha=1$

$$
\begin{aligned}
& c_{1}=\frac{p a^{-6}}{\frac{128}{7}+\frac{256}{49}}=0.042535 p a^{-6} \\
& \sigma_{y y}=-4 \times 0.042535 p a^{-6}\left(1-\frac{3 x^{2}}{a^{2}}\right)\left(1-\frac{y^{2}}{b^{2}}\right) a^{6} \\
& \sigma_{y y}=-0.1702 p\left(1-\frac{3 x^{2}}{a^{2}}\right)\left(1-\frac{y^{2}}{a^{2}}\right) \\
& \tau_{x y}=-0.68056\left(1-\frac{y^{2}}{a^{2}}\right)\left(1-\frac{x^{2}}{a^{2}}\right) \frac{x y}{a^{2}} \\
& \sigma_{x x}=p\left(1-\frac{y^{2}}{b^{2}}\right)-0.1702 p\left(1-\frac{x^{2}}{a^{2}}\right)^{2}\left(1-\frac{3 y^{2}}{a^{2}}\right)
\end{aligned}
$$

For a three term Ritz solution, we use

$$
\phi(x, y)=\phi_{0}+\left(c_{1}+c_{2} x^{2}+c_{3} y^{2}\right)\left(x^{2}-a^{2}\right)^{2}\left(y^{2}-b^{2}\right)^{2}
$$

The variational Ritz equations yield, on simplification,

$$
\begin{aligned}
& c_{1}\left(\frac{64}{7}+\frac{256}{49} \frac{b^{2}}{a^{2}}+\frac{64}{7} \frac{b^{4}}{a^{4}}\right)+c_{2} a^{2}\left(\frac{64}{77}+\frac{64}{49} \frac{b^{4}}{a^{4}}\right)+c_{3} a^{2}\left(\frac{64}{49} \frac{b^{2}}{a^{2}}+\frac{64}{77} \frac{b^{6}}{a^{6}}\right)=\frac{p}{a^{4} b^{2}} \\
& c_{1}\left(\frac{64}{11}+\frac{64}{7} \frac{b^{4}}{a^{4}}\right)+c_{2} a^{2}\left(\frac{192}{143}+\frac{256}{77} \frac{b^{2}}{a^{2}}+\frac{192}{7} \frac{b^{4}}{a^{4}}\right)+c_{3} a^{2}\left(\frac{64}{77} \frac{b^{2}}{a^{2}}+\frac{64}{77} \frac{b^{6}}{a^{6}}\right)=\frac{p}{a^{4} b^{2}} \\
& c_{1}\left(\frac{64}{7}+\frac{64}{11} \frac{b^{4}}{a^{4}}\right)+c_{2} a^{2}\left(\frac{64}{77}+\frac{64}{77} \frac{b^{4}}{a^{4}}\right)+c_{3} a^{2}\left(\frac{192}{7} \frac{b^{2}}{a^{2}}+\frac{256}{77} \frac{b^{4}}{a^{4}}+\frac{192}{143} \frac{b^{8}}{a^{6}}\right)=\frac{p}{a^{4} b^{2}}
\end{aligned}
$$

For square plates, $a=b$, and we obtain:

$$
\begin{aligned}
& 23.5102 c_{1}+2.1373 c_{2} a^{2}+2.1373 c_{3} a^{2}=\frac{p}{a^{6}} \\
& 14.9610 c_{1}+32.0959 c_{2} a^{2}+1.6623 c_{3} a^{2}=\frac{p}{a^{6}} \\
& 14.9610 c_{1}+1.6623 c_{2} a^{2}+32.0505 c_{3} a^{2}=\frac{p}{a^{6}}
\end{aligned}
$$

Solving, $c_{1}=0.04040 \mathrm{pa}^{-6}$

$$
c_{2}=c_{3}=0.01174 p a^{-8}
$$

Thus, $\phi(x, y)=\frac{p y^{2}}{2}\left(1-\frac{y^{2}}{6 b^{2}}\right)+0.04040 p a^{-6}\left(x^{2}-a^{2}\right)^{2}\left(y^{2}-b^{2}\right)^{2}$

$$
+(0.01174) p a^{-8}\left(x^{2}+y^{2}\right)\left(x^{2}-a^{2}\right)^{2}\left(y^{2}-b^{2}\right)^{2}
$$

Then, the stress fields are obtained as:

$$
\sigma_{x x}=\frac{\partial^{2} \phi}{\partial y^{2}}
$$




$$
\begin{aligned}
& \sigma_{x x}=p\left(1-\frac{y^{2}}{b^{2}}\right)+0.04040 p a^{-6}\left(x^{2}-a^{2}\right)^{2} \cdot 4\left(3 y^{2}-b^{2}\right) \\
& \quad+0.01174 p a^{-8}\left(x^{2}-a^{2}\right)^{2}\left(2 x^{2}-4 b^{2} x^{2}+30 y^{4}-24 b^{2} y^{2}+2 b^{4}\right) \\
& \sigma_{x x}=p\left(1-\frac{y^{2}}{b^{2}}\right)+0.1616 p a^{-6}\left(x^{2}-a^{2}\right)^{2}\left(3 y^{2}-b^{2}\right) \\
& \quad+0.01174 p a^{-8}\left(x^{2}-a^{2}\right)^{2}\left(2 x^{2}-4 b^{2} x^{2}+30 y^{4}-24 b^{2} y^{2}+2 b^{4}\right) \\
& \quad+0.01174 p a^{-4}\left(30 y^{4}-24 b^{2} y^{2}+2 b^{4}\right) \\
& \begin{array}{c}
\sigma_{x x}(x=0)=p\left(1-\frac{y^{2}}{b^{2}}\right)+0.1616 p a^{-6}\left(3 y^{2}-b^{2}\right) \\
\sigma_{y y}=\frac{\partial^{2} \phi}{\partial x^{2}}=0.1616 p a^{-2}\left(3 x^{2}-a^{2}\right) \\
+0.01174 p a^{-8}\left(y^{2}-b^{2}\right)^{2}\left(2 y^{2}-4 a^{2} x^{2}+30 x^{4}-24 a^{2} x^{2}+2 a^{4}\right) \\
\sigma_{y y}(x=0)=0.1616 p a^{-2}\left(-a^{2}\right)+0.01174 p a^{-8}\left(y^{2}-b^{2}\right)^{2}\left(2 y^{2}+2 a^{4}\right) \\
\sigma_{y y}(x=0)=-0.1616 p+0.01174 p a^{-8}\left(y^{2}-b^{2}\right)^{2}\left(2 y^{2}+2 a^{4}\right) \\
\sigma_{y y}(x=0, y=0)=-0.1616 p+0.01174 p a^{-8}\left(b^{4}\right) \cdot 2 a^{4} \\
\sigma_{y y}(x=0, y=0)=-0.1616 p+0.01174 p a^{-8} b^{4} \cdot 2 a^{4} \\
\sigma_{y y}(0,0)=-0.13812 p
\end{array}
\end{aligned}
$$

Thus, the distribution of normal stress $\sigma_{x x}$ on the cross-sectional plane $x=0$ is given by

$$
\sigma_{x x}(x=0, y)=p\left(1-\frac{y^{2}}{a^{2}}\right)-0.1616 p\left(1-\frac{3 y^{2}}{a^{2}}\right)+0.0235 p\left(1-\frac{12 y^{2}}{a^{2}}+\frac{15 y^{4}}{a^{4}}\right)
$$

The variation of the normal stress field $\sigma_{x x}$ on the plane $x=0$ for square plate is shown displayed in Table 1 for one term (parameter) Ritz solution, and in Table 2for three term (parameter) Ritz solution.

Table 1: One term (parameter) Ritz solution for distribution of normal stress $\sigma_{x x}$ on the plane $x=0$ for square plates.

\begin{tabular}{|l|l|}
\hline$y / a$ & $\sigma_{x x}(x=0)=p\left(1-\frac{y^{2}}{a^{2}}\right)-0.1702 p\left(1-\frac{3 y^{2}}{a^{2}}\right)$ \\
\hline 0 & $0.8298 p$ \\
\hline 0.2 & $0.8102 p$ \\
\hline 0.4 & $0.7515 p$ \\
\hline 0.6 & $0.6536 p$ \\
\hline 0.8 & $0.5166 p$ \\
\hline 1.0 & $0.3404 p$ \\
\hline
\end{tabular}

Table 2: Three term (parameter) Ritz solution for normal stress distribution on the plane $x=0$ for square plates.

\begin{tabular}{|l|l|}
\hline$y / a$ & $\sigma_{x x}(x=0)$ \\
\hline 0 & $0.8619 p$ \\
\hline 0.2 & $0.8306 p$ \\
\hline 0.4 & $0.7434 p$ \\
\hline 0.6 & $0.6206 p$ \\
\hline 0.8 & $0.4961 p$ \\
\hline 1.0 & $0.4172 p$ \\
\hline
\end{tabular}




\section{Discussion of Results}

The normal and shear stress distributions in a rectangular thin plate $2 a \times 2 b$ subjected to a parabolic distribution of loads on the two edges $x= \pm a$ have been found using the variational Ritz method. The given parabolic distribution of normal stress on the edges $x= \pm a$ was given as Equation (31), while the other edges were considered free of normal and shear stresses. The elastic stress analysis problem was formulated as a variational problem in terms of Airy's stress function; and the Ritz variational formulation presented as Equation (48). The problem was then solved by assuming that Airy's stress potential function can be approximated using a linear combination of coordinate shape (basis) functions that satisfy both the stress boundary conditions, as well as the domain conditions as given by Equation (42) - (45). For the parabolic distribution considered in the study, the Airy's stress potential function was found to be given by Equation (47). The problem was then solved for a one parameter and three parameter Airy's stress functions. A one parameter Airy's stress function yielded the unknown parameter given by Equation (72), and thus the solution for Airy's stress potential function as Equation (77). The normal and shear stresses were then determined from $\phi(x, y)$ as Equations (82), (91), and (94). The solutions were also presented for square plates as Equations (95) - (99) for a one parameter Ritz solution. The Airy's stress potential function considered for a three parameter Ritz solution was given as Equation (100). The variational Ritz equations yielded a system of three equations in the three unknown parameters of the Airy's stress functions and were given as Equations (101) - (103). The equations were solved for square plates to obtain the three parameters as Equations (107) and (108); hence the three parameter Airy's stress potential function for the problem was found as Equation (109). The stresses were found as Equations (111) and (114).

An examination of the normal stress distribution $\sigma_{x x}$ over the cross-section $x=0$ shows that as the plate aspect ratio increases i.e. $a / b \rightarrow 0$, the normal stress distribution over the cross-section $x=0$ becomes more uniform. For instance when $a / b=2$, the Airy's stress constants for a three parameter variational Ritz solution are $c_{1}=0.07983 p a^{-4} b^{-2}, c_{2}=0.1250 p a^{-6} b^{-2}$ and $c_{3}=0.01826 p a^{-6} b^{-2}$ and the distribution of $\sigma_{x x}$ over the cross-section, $x=0$ are given in Table 3 for $a / b=2$ for various values of $y / b$, yielding an average value of $\frac{2}{3} p$.

Table 3: Normal stress distribution over the cross-section $x=0$ for $a / b=2$

\begin{tabular}{|l|l|l|l|l|l|l|}
\hline$y / b$ & 0 & 0.2 & 0.4 & 0.6 & 0.8 & 1.0 \\
\hline$\sigma_{x x}(x=0)$ & $0.690 p$ & $0.684 p$ & $0.669 p$ & $0.653 p$ & $0.649 p$ & $0.675 p$ \\
\hline
\end{tabular}

The results of this study agree remarkably well with those presented in the technical literature.

\section{Conclusions}

The problem of finding stress fields in rectangular plates submitted to edge loads that are distributed non linearly on the edges of the plate is a classical problem of the theory of elasticity for which satisfactory closed form analytical solution are difficult to obtain. Hence, in this study, the Ritz variational method has been successfully applied to solve the elastic stress problem for a parabolic variation of normal stress $\sigma_{x x}$ on the two edges $x= \pm a$ of the plate. The problem was first formulated using variational principles of elasticity using the assumptions of linear elasticity, homogeneity and plane stress elasticity state. The total potential energy functional first expressed in stress terms was then formulated in terms of Airy's stress potential function $\phi(x, y)$. This had the advantage/merit of reducing the number of unknowns in the total potential energy functional from three stresses $\sigma_{x x}, \sigma_{x y}$ and $\tau_{x y}$ to one stress function $\phi(x, y)$. Ritz procedure was then applied to obtain the first variation of the total potential energy functional, yielding the Ritz variational functional or the Ritz variational equation. Following the Ritz variational method, the Airy's stress potential function was constructed to simultaneously satisfy both the stress boundary conditions along the four edges $x= \pm a$ and $y= \pm b$, as well as the biharmonic equation on the plate domain. This was achieved by using coordinate (shape) or basis functions that identically satisfy the biharmonic problem, as well as the edge boundary conditions. The Airy's stress functions assumed in terms of one unknown parameter, and in terms of three unknown parameters were used in this study. Upon substitution in Ritz variational equation, the problem simplified to an algebraic problem, leading to solutions of the unknown parameters. Hence, the Airy stress 
potential functions were completely determined. Thereafter, the normal stress fields and the shear stress fields were found from the Airy's stress functions. The solutions obtained for the normal and shear stress variations were found to be identical with solutions from the technical literature obtained using virtual stress principles. It was also found that as the plate aspect ratio $a / b$ increases, the normal stress distribution over the cross section of the plane $x=0$ becomes more uniform. Thus the following conditions can be made:

(i) The Ritz variational method is an effective approximate tool for the solution of elasticity problems of rectangular plates subjected to a parabolic distribution of edge loads in one direction.

(ii) A one parameter Ritz approximation of the Airy stress potential function in the variational equation yielded sufficiently accurate results for practical purposes.

(iii) As the plate aspect ratio increases, and the plate becomes very long in one direction relative to the other, the normal stress distribution over the cross section of the plane $x=0$ becomes uniform; a result that agrees with logical reasoning.

\section{References}

[1] Devarakonda K.K. (2004). Buckling and Flexural Vibration of Rectangular Plates Subjected to Half Sinusoidal Load on two opposite Edges. PhD Dissertation, School of Aerospace and Mechanical Engineering, Graduate Faculty, University of Oklahoma Graduate College 139pp.

[2] Dixit U.S. (2007). Finite Element Methods in Engineering (Lecture Notes). Department of Mechanical Engineering, Indian Institute of Technology, Guwahati Assam India, May 2007. Developed under the Curriculum Development Scheme of Quality Improvement Programme IIT Guwahati.

[3] Yang Yu-hua and Wang Xin-Wei (2011). Stress Analysis of thin Rectangular Plates under Non-linearly Distributed Edge Loads. Engineering Mechanics, Vol. 28, Issue 1, 37-042. 